\title{
Rapid classification of vehicle heading direction with two-axis magnetometer
}

\author{
Niklas Wahlström, Roland Hostettler, Fredrik Gustafsson and Wolfgang Birk
}

\section{Linköping University Post Print}

N.B.: When citing this work, cite the original article.

(C2012 IEEE. Personal use of this material is permitted. However, permission to reprint/republish this material for advertising or promotional purposes or for creating new collective works for resale or redistribution to servers or lists, or to reuse any copyrighted component of this work in other works must be obtained from the IEEE.

Niklas Wahlström, Roland Hostettler, Fredrik Gustafsson and Wolfgang Birk, Rapid classification of vehicle heading direction with two-axis magnetometer, 2012, Proceedings of the International Conference on Acoustics, Speech and Signal Processing (ICASSP), 33853388.

http://dx.doi.org/10.1109/ICASSP.2012.6288642

Postprint available at: Linköping University Electronic Press

http://urn.kb.se/resolve?urn=urn:nbn:se:liu:diva-85909 


\title{
RAPID CLASSIFICATION OF VEHICLE HEADING DIRECTION WITH TWO-AXIS MAGNETOMETER
}

\author{
Niklas Wahlström ${ }^{\dagger}$, Roland Hostettler ${ }^{\star}$, Fredrik Gustafsson ${ }^{\dagger}$, and Wolfgang Birk ${ }^{\star}$ \\ ${ }^{\dagger}$ Linköping University, Division of Automatic Control, Linköping, Sweden \\ *Luleå University of Technology, Division of Systems and Interaction, Luleå, Sweden \\ E-Mail: \{nikwa,fredrik\}@isy.liu.se, \{rolhos,wolfgang\}@ltu.se
}

\begin{abstract}
We present an approach for computing the heading direction of a vehicle by processing measurements from a 2-axis magnetometer rapidly. The proposed method relies on a non-linear transformation of the measurement data comprising only two inner products. Deterministic analysis of the signal model shows how the heading direction is contained in the signal and the proposed estimator is analyzed in terms of its statistical properties. Experimental verification indicates that good performance is achieved under the presence of saturation, measurement noise, and near field effects.
\end{abstract}

Index Terms - magnetometer, heading direction

\section{INTRODUCTION}

Existing methods for traffic monitoring such as inductive loops are more and more challenged by emerging solutions based on smallsize, low-cost sensors such as microphones or magnetometers. These sensors are often incorporated in sensor nodes for wireless sensor networks (WSN). One of the biggest advantages of using this technology is its flexibility. Nodes can be easily placed at points of interest and due to their connectivity, the measurement data can be made available almost instantly. Furthermore, the low cost makes them very attractive $[1,2]$.

However, such sensor nodes also bring certain limitations. Generally, the energy budget is limited as the units are powered by either batteries or solar panels [3]. Furthermore, computational resources are limited, either for the reason of power saving (e.g. duty-cycling of the computations or low-power processors) or due to sharing of the microcontroller between different tasks (measuring, computing, communication, etc.), see, e.g. [4, 5]. Thus, it is very important that the computation time for each task is reduced to a minimum, which emphasizes the need for low-complexity data processing algorithms.

One of the quantities of interest e.g. for road administrations, urban planners, or traffic management centers is traffic flow and, associated with that, the heading direction. The latter is important because it provides more insightful information regarding the traffic flow direction. With this information, the traffic volume that is normally measured by a simple detector can be analyzed more thoroughly and better conclusions for future measures such as road planning can be drawn.

In this paper, a scheme for classifying the heading direction of a vehicle in a fast and efficient way using measurements from a single

This work has been supported by the Swedish Foundation for Strategic Research under the project Cooperative Localization, the Swedish Governmental Agency for Innovation Systems (VINNOVA), and GEVEKO AB.
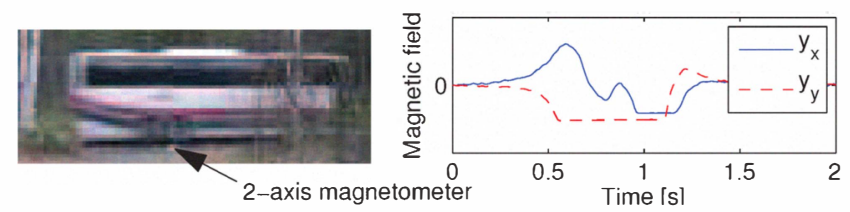

Fig. 1: A metallic vehicle gives rise to a magnetic field distortion. How do we, in a computationally cheap way, determine the heading direction from this information?

sensor node is proposed. The problem is stated in Section 2, followed by the development of the algorithm in Section 3. Finally, the method is applied to measurement data from commercially available sensors and evaluated in Section 4.

\section{PROBLEM FORMULATION}

When a metallic target passes, a stationary magnetometer measures a distortion of the magnetic field [6]. This distortion contains rich information which is associated with both the target trajectory as well as target specific parameters. One example of such a distortion is shown in Figure 1, where two components of the magnetic field are measured.

In this work we are only interested in finding the correct heading direction of the target. Consequently, the method should be insensitive to other quantities such as velocity, distance between the sensor and the trajectory, magnetic signature, and target extension. Additionally, the following physical limitations of the sensor should be taken into consideration: a) The magnetometer has only two axes: This means that the magnetometer only measures the two components of the magnetic field parallel to the ground. and not the third component orthogonal to the ground. b) The signal might be saturated: Especially when large targets pass close to the sensor, the measured distortion is saturated as shown in Figure 1. c) Measurement noise: The magnetometer measures the magnetic field disturbed by noise.

One way of solving the problem is to approximately model the target as a magnetic dipole. This approximation holds if the distance between the target and the sensor is large in comparison to the characteristic length of the target. This gives raise to a magnetic dipole field $\mathbf{h}(t)$ expressed as

$$
\mathbf{h}(t)=\frac{3(\mathbf{r}(t) \cdot \mathbf{m}) \mathbf{r}(t)-\|\mathbf{r}(t)\|^{2} \mathbf{m}}{\|\mathbf{r}(t)\|^{5}},
$$

where $\mathbf{r}(t)$ is the position of the target relative to the sensor and $\mathbf{m}$ is the magnetic dipole moment, which can be considered as a target 


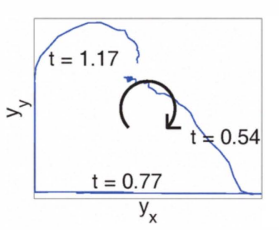

(a)

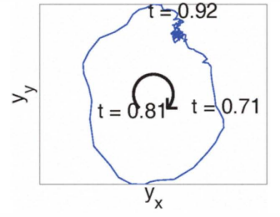

(b)

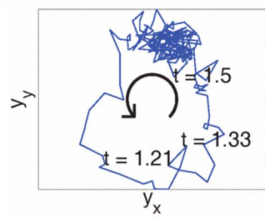

(c)
Fig. 2: Measurement trajectories for three different vehicles. (a) and (b) Vehicles passing from left to right, (c) vehicle passing from right to left.

specific parameter [7]. Two components of the magnetic field (1) can then be measured with a two-axis magnetometer

$$
\mathbf{y}(k T)=\left[\begin{array}{lll}
1 & 0 & 0 \\
0 & 1 & 0
\end{array}\right] \mathbf{h}(k T)+\mathbf{e}(k T), \quad k=1, \cdots, N,
$$

where $T$ is the sampling time of the sensor and $\mathbf{e}(k T)$ measurement noise. The models (1) and (2) can now be used to estimate $\mathbf{r}(t)$ from the measurement of $\mathbf{y}(k T)$ and the heading information can be extracted from the estimated trajectory $\hat{\mathbf{r}}(t)$. However, this is a non-linear problem and convergence is not guaranteed.

Furthermore, if the target is close to the sensor, a higher order model including more parameters is needed to describe the signal accurately, for example by including higher order moments of the magnetic field or by modeling the target as a grid of dipoles [8]. Unfortunately, the computational cost of the corresponding estimation problem would grow quadratically with the number of parameters.

Instead of using a model based approach, we will, with (1) as basis, construct a test statistic from the magnetometer signal directly. The sign of this test statistic will be shown to be sensitive to the heading direction of the target.

\section{HEADING DIRECTION INFORMATION}

The heading direction information of the two dimensional magnetometer signal in Figure 1 can partly be revealed by plotting the two dimensions of the measurement against each other in a graph as presented in Figure 2a. As it will be shown, the measurement trajectory is turning clockwise in the $x$ - $y$-plane as time increases. Further, it is easy to realize that the measurement trajectory would turn counterclockwise if the same vehicle reversed in the opposite direction. This can be seen by changing the time direction for the measurement sequence.

Consequently, the rotation direction of the measurement trajectory might indicate the heading direction of the target. The equivalent plot can be made for other vehicles, and, according to Figures $2 b$ and $2 c$, this pattern is recognized for them as well.

Before proving this relation, we will simulate the dipole model (1) for a predefined trajectory to get even more intuition. This is done in the following example.

\subsection{Example}

Assume a linear trajectory orthogonal to the $z$-direction $\mathbf{r}(t)=$ $[t, 1,0]^{T}$ and a magnetic dipole moment $\mathbf{m}=\left[m^{x}, m^{y}, m^{z}\right]^{T}$. From (1) we get

$$
\mathbf{h}(t)=\frac{1}{{\sqrt{t^{2}+1}}^{5}}\left[\begin{array}{c}
2 m^{x} t^{2}+3 m^{y} t-m^{x} \\
-m^{y} t^{2}+3 m^{x} t+2 m^{x} \\
\left(t^{2}+1\right) m^{z}
\end{array}\right] .
$$

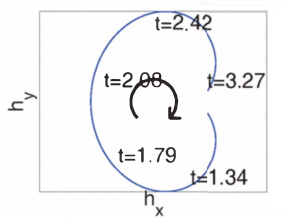

(a)

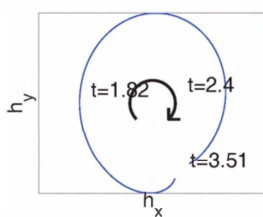

(b)

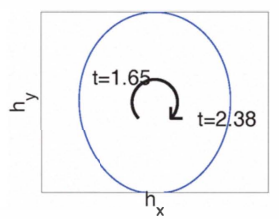

(c)
Fig. 3: Simulated measurement trajectories for vehicles moving from left to right with (a) $\mathbf{m}=[1,0,0]^{T}$, (b) $\mathbf{m}=[1,1,0]^{T}$, (c) $\mathbf{m}=[0,1,0]^{T}$.

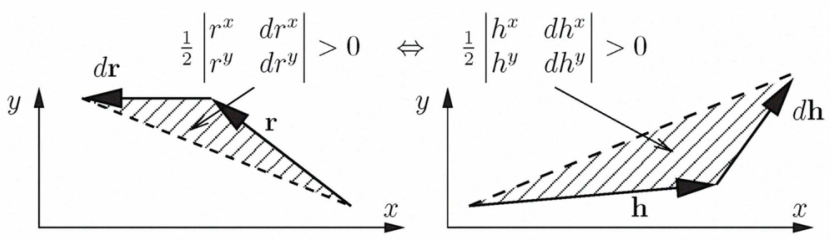

Fig. 4: Graphical illustration of Theorem 1.

By plotting the $x$ and $y$ components of $\mathbf{h}(t)$ against each other for different values of $\mathbf{m}$, we conclude that all trajectories of $\mathbf{h}(t)$ turn clockwise (see Figure 3).

Furthermore, from (3) we observe that the $h^{z}(t)$ does not have any heading direction information since it is an even function. Thus, since the magnetometer we are using in this work only measures the $x$ and $y$ components of the magnetic field, the exclusion of the $z$ component will not lead to any information loss regarding the heading direction.

\subsection{Deterministic analysis}

Next, we will prove that the conclusion from the example will hold for all $\mathbf{m}$ and arbitrary trajectories as long as they lie in the $x-y$ plane. The proof is accomplished by computing the area that the target trajectory and the measurement trajectory are enclosing. These two areas will be proved to have the same sign. For an infinitesimal position change $d \mathbf{r}$ this area equalizes the area of the triangle that $\mathbf{r}$ and $d \mathbf{r}$ are spanning, see Figure 4 . From linear algebra it is known that this area can be computed with the determinant of the $2 \times 2$ matrix, whose columns consist of the vectors spanning the triangle.

The following theorem shows that this area has the same sign for the target trajectory as for the measurement trajectory. Consequently, if the target trajectory is turning clockwise around the sensor, the trajectory of the magnetic field $\mathbf{h}(t)$ will also turn clockwise and vice versa. The theorem is also illustrated in Figure 4.

Theorem 1. Assume the magnetic dipole model

$$
\mathbf{h}=\frac{3(\mathbf{r} \cdot \mathbf{m}) \mathbf{r}-\|\mathbf{r}\|^{2} \mathbf{m}}{\|\mathbf{r}\|^{5}}
$$

Let $\mathbf{r}=\left[r^{x}, r^{y}, 0\right]^{T}, d \mathbf{r}=\left[d r^{x}, d r^{y}, 0\right]^{T}$ and $\mathbf{m}=\left[m^{x}, m^{y}, m^{z}\right]^{T}$. Then,

$$
\left|\begin{array}{ll}
h^{x} & d h^{x} \\
h^{y} & d h^{y}
\end{array}\right|=\beta\left|\begin{array}{ll}
r^{x} & d r^{x} \\
r^{y} & d r^{y}
\end{array}\right|, \quad \beta>0
$$

where

$$
\left[h^{x}, h^{y}, h^{z}\right]^{T}:=\mathbf{h} \quad \text { and } \quad\left[d h^{x}, d h^{y}, d h^{z}\right]^{T}:=d \mathbf{h}=\frac{\partial \mathbf{h}}{\partial \mathbf{r}} d \mathbf{r} .
$$


Proof. Starting from (4) we can compute

$$
\begin{aligned}
d \mathbf{h}=\frac{\partial \mathbf{h}}{\partial \mathbf{r}} d \mathbf{r}= & \frac{3}{\|\mathbf{r}\|^{5}}((\mathbf{r} \cdot \mathbf{m}) d \mathbf{r}+(\mathbf{m} \cdot d \mathbf{r}) \mathbf{r} \\
& \left.+(\mathbf{r} \cdot d \mathbf{r}) \mathbf{m}-5 \frac{(\mathbf{r} \cdot \mathbf{m})(\mathbf{r} \cdot d \mathbf{r})}{(\mathbf{r} \cdot \mathbf{r})} \mathbf{r}\right) .
\end{aligned}
$$

Now, define the notation of a pair of two orthogonal 2-dimensional vectors from $\mathbf{u} \in \mathbb{R}^{3}$

$$
\overline{\mathbf{u}}=\left[u^{x}, u^{y}\right]^{T} \quad \text { and } \quad \overline{\mathbf{u}}_{\perp}=\left[u^{y},-u^{x}\right]^{T},
$$

where $\mathbf{u}=\left[u^{x}, u^{y}, u^{z}\right]^{T}$. Furthermore, for $\overline{\mathbf{u}}, \overline{\mathbf{v}}, \overline{\mathbf{w}} \in \mathbb{R}^{2}$ we can easily verify the following identities

$$
\begin{aligned}
\left(\overline{\mathbf{u}} \cdot \overline{\mathbf{v}}_{\mathbf{\Lambda}}\right) & =-\left(\overline{\mathbf{u}}_{\mathbf{\Lambda}} \cdot \overline{\mathbf{v}}\right) \\
(\overline{\mathbf{u}} \cdot \overline{\mathbf{u}})(\overline{\mathbf{v}} \cdot \overline{\mathbf{w}}) & =(\overline{\mathbf{v}} \cdot \overline{\mathbf{u}})(\overline{\mathbf{u}} \cdot \overline{\mathbf{w}})+\left(\overline{\mathbf{v}} \cdot \overline{\mathbf{u}}_{\mathbf{\Lambda}}\right)\left(\overline{\mathbf{u}}_{\mathbf{\Lambda}} \cdot \overline{\mathbf{w}}\right) .
\end{aligned}
$$

In addition, we also notice that a $2 \times 2$ determinant can be rewritten as an inner product

$$
\left|\begin{array}{ll}
u^{x} & v^{x} \\
u^{y} & v^{y}
\end{array}\right|=\left(\overline{\mathbf{u}} \cdot \overline{\mathbf{v}}_{\mathbf{\Lambda}}\right)
$$

Since $r^{z}=0$ and $d r^{z}=0$, the two-dimensional version of (4) and (6) can now easily be stated as

$$
\begin{gathered}
\overline{\mathbf{h}}=\frac{3(\overline{\mathbf{r}} \cdot \overline{\mathbf{m}}) \overline{\mathbf{r}}-\|\overline{\mathbf{r}}\|^{2} \overline{\mathbf{m}}}{\|\overline{\mathbf{r}}\|^{5}} \\
d \overline{\mathbf{h}}=\frac{3}{\|\overline{\mathbf{r}}\|^{5}}((\overline{\mathbf{r}} \cdot \overline{\mathbf{m}}) d \overline{\mathbf{r}}+(\overline{\mathbf{m}} \cdot d \overline{\mathbf{r}}) \overline{\mathbf{r}} \\
\left.+(\overline{\mathbf{r}} \cdot d \overline{\mathbf{r}}) \overline{\mathbf{m}}-5 \frac{(\overline{\mathbf{r}} \cdot \overline{\mathbf{m}})(\overline{\mathbf{r}} \cdot d \overline{\mathbf{r}})}{(\overline{\mathbf{r}} \cdot \overline{\mathbf{r}})} \overline{\mathbf{r}}\right) .
\end{gathered}
$$

Combining (10) and the identities (8) yields

$$
\begin{array}{r}
\frac{\|\overline{\mathbf{r}}\|^{10}}{3}(\overline{\mathbf{h}} \cdot d \overline{\mathbf{h}} \mathbf{\perp})=3(\overline{\mathbf{r}} \cdot \overline{\mathbf{m}})^{2}\left(\overline{\mathbf{r}} \cdot d \overline{\mathbf{r}}_{\mathbf{\Lambda}}\right) \\
\underbrace{+(\overline{\mathbf{r}} \cdot \overline{\mathbf{m}})(\overline{\mathbf{r}} \cdot d \overline{\mathbf{r}})\left(\overline{\mathbf{m}} \cdot \overline{\mathbf{r}}_{\mathbf{\Lambda}}\right)-\|\overline{\mathbf{r}}\|^{2}(\overline{\mathbf{m}} \cdot \overline{\mathbf{r}})\left(\overline{\mathbf{m}} \cdot d \overline{\mathbf{r}}_{\perp}\right)}_{+\left(\overline{\mathbf{r}} \cdot \overline{\mathbf{m}}_{\perp}\right)^{2}\left(\overline{\mathbf{r}} \cdot d \overline{\mathbf{r}}_{\perp}\right)} \\
\underbrace{+(\overline{\mathbf{r}} \cdot \overline{\mathbf{m}})(\overline{\mathbf{r}} \cdot d \overline{\mathbf{r}})\left(\overline{\mathbf{m}} \cdot \overline{\mathbf{r}}_{\mathbf{\Lambda}}\right)-\|\overline{\mathbf{r}}\|^{2}(\overline{\mathbf{m}} \cdot d \overline{\mathbf{r}})\left(\overline{\mathbf{m}} \cdot \overline{\mathbf{r}}_{\perp}\right)}_{-(\overline{\mathbf{r}} \cdot \overline{\mathbf{m}})^{2}\left(\overline{\mathbf{r}} \cdot d \overline{\mathbf{r}}_{\perp}\right)} \\
=\left[2(\overline{\mathbf{r}} \cdot \overline{\mathbf{m}})^{2}+\left(\overline{\mathbf{r}} \cdot \overline{\mathbf{m}}_{\perp}\right)^{2}\right]\left(\overline{\mathbf{r}} \cdot d \overline{\mathbf{r}}_{\perp}\right) .
\end{array}
$$

Finally, by using the relation (9), we will arrive at the result in (5) with

$$
\beta:=\frac{3}{\|\mathbf{r}\|^{10}}\left[2(\overline{\mathbf{r}} \cdot \overline{\mathbf{m}})^{2}+\left(\overline{\mathbf{r}} \cdot \overline{\mathbf{m}}_{\mathbf{\Lambda}}\right)^{2}\right]>0 .
$$

From Theorem 1 we can now define a indicator for the heading direction based on the magnetic field components by integrating over all infinitesimal area segments

$$
f=\int\left|\begin{array}{ll}
h^{x} & d h^{x} \\
h^{y} & d h^{y}
\end{array}\right|=\int\left|\begin{array}{ll}
h^{x}(t) & d h^{x}(t) / d t \\
h^{y}(t) & d h^{y}(t) / d t
\end{array}\right| d t .
$$

Finally, the discrete time version of (12) will then be

$$
f^{T}=\sum_{k=1}^{N-1}\left|\begin{array}{ll}
h_{k}^{x} & \left(h_{k+1}^{x}-h_{k}^{x}\right) / T \\
h_{k}^{y} & \left(h_{k+1}^{y}-h_{k}^{y}\right) / T
\end{array}\right| T=\sum_{k=1}^{N-1}\left(h_{k}^{x} h_{k+1}^{y}-h_{k}^{y} h_{k+1}^{x}\right) .
$$

where the notation $h_{k}^{x}=h^{x}(k T)$ has been used.

\subsection{Stochastic analysis}

So far the magnetic field is assumed to be known deterministically. Therefor, we will investigate how measurement noise will affect the heading direction estimation by considering the twodimensional measurement equation (2) , where the measurement noise is assumed to be normal distributed $\mathbf{e}_{k} \sim$ $\mathcal{N}\left(\mathbf{0}, \sigma^{2} \mathbf{I}_{2}\right)$. Based on the result from the last section a decent estimator of (13) would then be

$$
\hat{f}^{T}=\sum_{k=1}^{N-1}\left(y_{k}^{x} y_{k+1}^{y}-y_{k}^{y} y_{k+1}^{x}\right) \text {. }
$$

By using (2), (13) and (14), the estimator (14) can easily be shown to be unbiased. Further, the variance of (14) can be computed as

$$
\operatorname{Var}\left(\hat{f}^{T}\right)=4 \sigma^{2} \sum_{k=1}^{N-1}\left\|\Delta \overline{\mathbf{h}}_{k}\right\|^{2}+2 \sigma^{4}(N-1),
$$

where $\Delta \overline{\mathbf{h}}_{k} \approx\left(\overline{\mathbf{h}}_{k+1}-\overline{\mathbf{h}}_{k-1}\right) / 2$. Consequently, the variance of the estimator scales with $\sigma^{4}$, which makes it very sensitive to noise. However, its impact can be reduced to $\sigma^{2}$ by modifying the estimator such that it does not sum over each individual area contribution from two consecutive samples, but rather average over larger area segments, see Figure 5. In this case, however, each individual segment between $k$ and $k+1$ will be counted $p$ times. Thus, the estimator has to be normalized by $1 / p$ so that its value still has the interpretation of being the enclosed area.

$$
\hat{f}_{p}^{T}=\frac{1}{p} \sum_{k=1}^{N-p}\left(y_{k}^{x} y_{k+p}^{y}-y_{k}^{y} y_{k+p}^{x}\right) .
$$

This modified estimator will be slightly biased, however, it will have a lower variance, which can be computed as

$$
\operatorname{Var}\left(\hat{f}_{p}^{T}\right)=4 \sigma^{2} \sum_{k=1}^{N-p}\left\|\Delta \overline{\mathbf{h}}_{k}\right\|^{2}+\frac{2 \sigma^{4}(N-p)}{p^{2}},
$$

where $\Delta \overline{\mathbf{h}}_{k} \approx\left(\overline{\mathbf{h}}_{k+p}-\overline{\mathbf{h}}_{k-p}\right) / 2 p$. Now, as long as

$$
\frac{2 \sigma^{2}(N-p)}{p^{2}} \gg 4 \sum_{k=1}^{N-p}\left\|\Delta \overline{\mathbf{h}}_{k}\right\|^{2}
$$

the first term in (17) will dominate. By choosing

$$
p \gg \frac{\sigma / 2}{\sqrt{\frac{1}{N-p} \sum_{k=1}^{N-p}\left\|\Delta \overline{\mathbf{h}}_{k}\right\|^{2}}},
$$

the variance of the estimator will only scale with $\sigma^{2}$

$$
\operatorname{Var}\left(\hat{f}_{p}^{T}\right) \approx 4 \sigma^{2} \sum_{k=1}^{N-p}\left\|\Delta \overline{\mathbf{h}}_{k}\right\|^{2}
$$

However, we might not choose $p$ a too large since the increasing bias of (16) will deteriorate the classification results.

Finally, the improved estimator (16) can be written as the difference of the two inner products

$$
\hat{f}_{p}^{T}=\left(\mathbf{Y}_{1:(N-p)}^{x}\right)^{T} \mathbf{Y}_{(1+p): N}^{y}-\left(\mathbf{Y}_{1:(N-p)}^{y}\right)^{T} \mathbf{Y}_{(1+p): N}^{x}
$$

where $\mathbf{Y}_{m: n}^{\alpha}=\left[y_{m}^{\alpha}, y_{m+1}^{\alpha}, \cdots, y_{n-1}^{\alpha}, y_{n}^{\alpha}\right]^{T}$. 


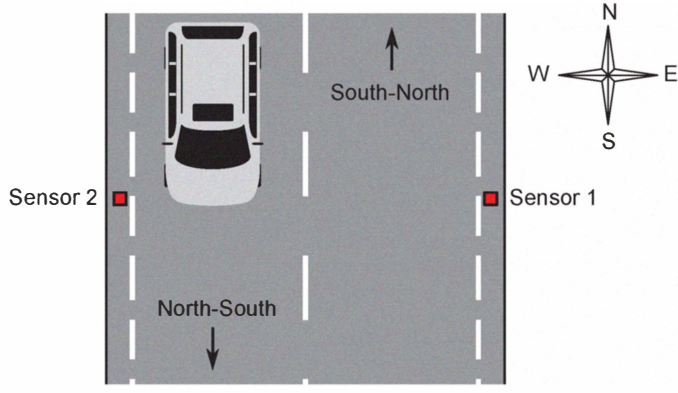

Fig. 6: Illustration of the experiment setup showing the two sensors on each side of the road as well as the heading directions.

Table 1: Results of applying the heading direction classification to the measurement data. For example, 87 out of the 88 vehicles travelling south-north were classified correctly by the measurements of sensor 1 .

\begin{tabular}{lcc}
\hline & $\begin{array}{c}\text { South-North } \\
\text { (Sensor 1) }\end{array}$ & $\begin{array}{c}\text { North-South } \\
\text { (Sensor 2) }\end{array}$ \\
\hline Sensor 1 & $87 / 88$ & $91 / 99$ \\
Sensor 2 & $82 / 88$ & $99 / 99$ \\
\hline
\end{tabular}

\section{EXPERIMENTAL RESULTS}

\subsection{Experiment setup}

Experiments were conducted in order to verify the proposed algorithm on real data. The measurements were performed on a two-way country road with moderate traffic density. Commercially available 2-axis magnetometers (Honeywell HMC6042, [9]) including the corresponding signal conditioning and acquisition hardware sampling at $100 \mathrm{~Hz}$ were placed on both sides of the road as illustrated in Figure 6. Traffic was measured for 45 minutes and 88 vehicles travelling south-north (close to sensor 1) and 99 vehicles travelling north-south (close to sensor 2) were captured. Furthermore, the whole experiment was recorded with a video camera.

From the video recording the true passing time of the vehicles as well as their heading directions were manually extracted. For each passage a $1.0 \mathrm{~s}$ long time window from the magnetometer signal was extracted and the heading direction was classified with the method described in Section 3 with $p=10$, which was the value maximizing the classification performance. The classification was then compared to the ground truth. Note that the detection problem is not considered here. However, this could be done, for example by using methods described in [2].

\subsection{Results and discussion}

The results are summarized in Table 1 . It indicates that the sensors are capable of classifying the heading directions of the vehicles passing in the nearby lane with a very high accuracy with almost no wrong classification. Furthermore, most of the vehicles passing on the distant lane are also classified correctly.

Even though the statistical significance of these results is low due to low number of samples, they clearly show that the proposed method can correctly classify the heading direction of vehicles in the close vicinity of the sensor. Consequently, the proposed method is robust even if near field effects are present. Furthermore, the vehicles farther away are more difficult to classify due to worse SNR.
Additionally, if two vehicles meet each other in front of the sensors, each sensor will most likely classify the heading direction of the vehicle passing on the distant lane wrongly. In total four missclassifications in the results can be explained this way. However, this problem can be solved by fusing the information from the two sensors.

Finally, the ability of the proposed method to handle saturation can intuitively be understood by considering its geometrical interpretation as being the enclosed area of the measurement trajectory, as described in Section 3. A saturation will only crop this area but not change its sign, see for example Figure 2a.

\section{CONCLUSION}

In this paper, a technique for fast and efficient vehicle heading classification using a two-axis magnetometer has been proposed. It was shown theoretically and by applying the method to measurement data from commercially available sensors with certain limitations (namely saturation), that the proposed algorithm works well if the SNR is high enough, even though the simplified dipole model in (1) was assumed to be the general case. However, the method has difficulties of handling multiple vehicles in front of the sensor.

Future work should focus on verifying the method more systematically, including the analysis of how the near-field effects, where the single dipole-model is a very crude approximation, affect the algorithm. Furthermore, more real world data tests as well as the implementation in a real sensor platform should be targeted.

\section{REFERENCES}

[1] W. Birk, E. Osipov, and J. Eliasson, "iRoad - cooperative road infrastructure systems for driver support," in 16th ITS World Congress 2009, Stockholm Sweden, September 2009.

[2] S.-Y. Cheung and P. Varaiya, "Traffic surveillance by wireless sensor networks: Final report," California Path Program, Institute of Transportation Studies, University of California, Berkeley, Tech. Rep., 2007.

[3] G. Girban and M. Popa, "A glance on WSN lifetime and relevant factors for energy consumption," in Computational Cybernetics and Technical Informatics (ICCC-CONTI), 2010 International Joint Conference on, may 2010, pp. $523-528$.

[4] S. Giannecchini, M. Caccamo, and C.-S. Shih, "Collaborative resource allocation in wireless sensor networks," in Real-Time Systems, 2004. ECRTS 2004. Proceedings. 16th Euromicro Conference on, June 2004, pp. 35-44.

[5] Y. Yu and V. K. Prasanna, "Energy-balanced task allocation for collaborative processing in wireless sensor networks," Mobile Networks and Applications, vol. 10, pp. 115-131, 2005.

[6] Honeywell, "Vehicle detection using AMR sensors," Honeywell International Inc., Tech. Rep., 2005.

[7] N. Wahlström, J. Callmer, and F. Gustafsson, "Magnetometers for tracking metallic targets," in Proceedings of 13th International Conference on Information Fusion, Edinburgh, Scotland, July 2010.

[8] W. M. Wynn, "Detection, localization, and characterization of static magnetic dipole sources," in Detection and Identification of Visually Obscured Targets. Taylor and Francis, 1999, pp. 337-374.

[9] Honeywell, 2-Axis Magnetic Sensor Circuit HMC6042, datasheet. 